\title{
High-pressure phases and transitions of the layered alkaline earth nitridosilicates $\mathrm{SrSiN}_{2}$ and $\mathrm{BaSiN}_{2}$
}

\author{
S Rebecca Römer ${ }^{1}$, Peter Kroll ${ }^{2}$ and Wolfgang Schnick ${ }^{1}$ \\ ${ }^{1}$ Department Chemie und Biochemie, Lehrstuhl für Anorganische Festkörperchemie, \\ Ludwig-Maximilians-Universität München, Butenandtstraße 5-13 (D), D-81377 München, \\ Germany \\ ${ }^{2}$ Department of Chemistry and Biochemistry, The University of Texas at Arlington, \\ 700 Planetarium Place Arlington, TX 76019-0065, USA \\ E-mail: wolfgang.schnick@uni-muenchen.de
}

Received 25 February 2009, in final form 28 April 2009

Published 12 June 2009

Online at stacks.iop.org/JPhysCM/21/275408

\begin{abstract}
We investigate the high-pressure phase diagram of $\mathrm{SrSiN}_{2}$ and $\mathrm{BaSiN}_{2}$ with density-functional calculation. Searching a manifold of possible candidate structures, we propose new structural modifications of $\mathrm{SrSiN}_{2}$ and $\mathrm{BaSiN}_{2}$ attainable in high-pressure experiments. The monoclinic ground state of $\mathrm{SrSiN}_{2}$ transforms at $3 \mathrm{GPa}$ into an orthorhombic $\mathrm{BaSiN}_{2}$ type. At $14 \mathrm{GPa}$ a $\mathrm{CaSiN}_{2}$-type structure becomes the most stable configuration of $\mathrm{SrSiN}_{2}$. A hitherto unknown $\mathrm{Pbcm}$ modification is adopted at $85 \mathrm{GPa}$ and, finally, at $131 \mathrm{GPa}$ a $\mathrm{LiFeO}_{2}$-type structure. The higher homologue $\mathrm{BaSiN}_{2}$ transforms to a $\mathrm{CaSiN}_{2}$ type at $41 \mathrm{GPa}$ and further to a $\mathrm{Pbcm}$ modification at $105 \mathrm{GPa}$. Both systems follow the pressure-coordination rule: the coordination environment of $\mathrm{Si}$ increases from tetrahedral through trigonal bipyramidal to octahedral. Some high-pressure phases are related in structure through simple group-subgroup mechanisms, indicating displacive phase transformations with low activation barriers.
\end{abstract}

S] Supplementary data are available from stacks.iop.org/JPhysCM/21/275408

\section{Introduction}

In our continuing interest in nitridosilicates, their structural modifications and potential high-pressure phases, we investigate $\mathrm{SrSiN}_{2}$ and $\mathrm{BaSiN}_{2}$. Previously we reported on $\mathrm{BeSiN}_{2}$, $\mathrm{MgSiN}_{2}$ and $\mathrm{CaSiN}_{2}$ [1]. Their ground state modifications resemble those of stuffed $\beta$-cristobalite variants. The structures with the smaller alkaline earth ions $(\mathrm{Be}, \mathrm{Mg}$ ) can alternatively be described as ordered wurtzite structures. High-pressure phases of $\mathrm{BeSiN}_{2}, \mathrm{MgSiN}_{2}$ and $\mathrm{CaSiN}_{2}$ are proposed to adopt chalcopyrite-type structures and, finally, adopt ordered $\mathrm{NaCl}$ super-structures with sixfold coordination of Si.

$\mathrm{SrSiN}_{2}$ and $\mathrm{BaSiN}_{2}$ [2], on the contrary, differ considerably from the other three $\mathrm{MSiN}_{2}$ compounds. While they comprise $\mathrm{SiN}_{4}$ tetrahedra as well, these tetrahedra do not form three-dimensional networks. Instead, layers are formed by connecting 'bowtie' units $\mathrm{Si}_{2} \mathrm{~N}_{6}$ (pairs of edge-sharing tetrahedra) through common corners. $\mathrm{BaSiN}_{2}$ crystallizes in the orthorhombic space group Cmca (no 64), whereas $\mathrm{SrSiN}_{2}$ adopts a monoclinic distorted variant $\left(P 2_{1} / c\right.$, no 14$)$ of the same structure. The different structural behaviour at ambient pressure may be attributed to the larger size of the alkaline earth ion. An interesting question is how such structures may behave if high pressure is applied.

In this work, we report the optimized geometries of ambient pressure $\mathrm{SrSiN}_{2}$ and $\mathrm{BaSiN}_{2}$. We further set out to investigate candidate high-pressure phases by scanning over a manifold of $\mathrm{ABX}_{2}$ structures. Anticipating our results, we will predict four high-pressures phases of $\mathrm{SrSiN}_{2}$ and two for $\mathrm{BaSiN}_{2}$, with $\mathrm{SrSiN}_{2}$ showing the first transition as early as $3 \mathrm{GPa}$. We will also address possible mechanism of the encountered phase transformations on the basis of groupsubgroup relations.

\section{Methods}

Structural optimizations, total energies, and properties are calculated within density-functional theory (DFT) [3], for 
which we use a standard plane-wave/pseudopotential approach as implemented in the Vienna $a b$ initio simulation package (VASP) [4]. The generalized gradient approximation (GGA) [5] together with the projector-augmented-wave (PAW) [6] method is employed to accurately compute relative enthalpies between structures with different coordination environments. For comparison, we also computed all structures using the local density approximation (LDA). An energy cut-off of $500 \mathrm{eV}$ for the expansion of the wavefunction into the plane-wave basis is chosen. Appropriate $k$-point grids (see table A1 in supplementary data available at stacks.iop.org/JPhysCM/21/275408) according to the Monkhorst-Pack scheme [7] facilitate Brillouin-zone integration.

Candidates for high-pressure phases of the $\mathrm{ABX}_{2}$ type were collected from the ICSD [8]. In total, we investigated more than 15 hypothetical polymorphs of $\mathrm{MSiN}_{2}$. All structural parameters, positions as well as cell parameters, are then optimized for the given composition. Convergence is achieved when residual forces are below $5 \times 10^{-3} \mathrm{eV}^{-1}$. The symmetry after structure optimization is checked via the subroutine ADDSYM in the program PLATON [9]. Complete crystallographic data of the optimized positions for all presented structures are given in the supplementary data (available at stacks.iop.org/JPhysCM/21/275408).

Pressure is simulated by changing the volume of the cell and optimizing the structure again under the constraint of constant volume. The resulting energy-volume $(E-V)$ graph is fitted with the Murnaghan equation of state (EOS) [10] to yield the bulk modulus $B_{0}$. We obtain further the pressure from the $E-V$ graph by numerical differentiation of a Murnaghan EOS fit to the data, $p=-\partial E / \partial V$. The enthalpy $H$ of a structure is calculated via $H=E+p V$. We neglect entropy contributions between solid-state structures, making the enthalpy difference $\Delta H$ the measure to identify phase transformations. This approach is justified, because entropy differences between solid-state crystal structures are typically small in comparison to changes of $\Delta H$ within $1 \mathrm{GPa}$ of pressure change.

\section{Results}

\section{1. $\mathrm{SrSiN}_{2}$}

$\alpha-\mathrm{SrSiN}_{2}$ crystallizes with monoclinic space group $P 2_{1} / c$ (no 14) in a layered structure [2] (figure 1). The basic structural motifs are pairs of edge-sharing $\mathrm{SiN}_{4}$ tetrahedra, which are connected further through corners to other tetrahedra pairs (figure 1). $\mathrm{Sr}^{2+}$ ions are located between the layers, with each $\mathrm{Sr}^{2+}$ ion eightfold coordinated by N. Noteworthily, the monoclinic structure of $\alpha-\mathrm{SrSiN}_{2}$ is a distortion of the more symmetric orthorhombic structure of $\mathrm{BaSiN}_{2}$ (space group Cmca, no 64 [2], see also figure 1).

The results of our calculations-phase diagrams of $\mathrm{SrSiN}_{2}$ - are given in figure 2. Note that we present energyvolume and enthalpy-pressure data for the relevant structures only. In total we computed more than a dozen candidates. According to figure 2, the first structural transition appears
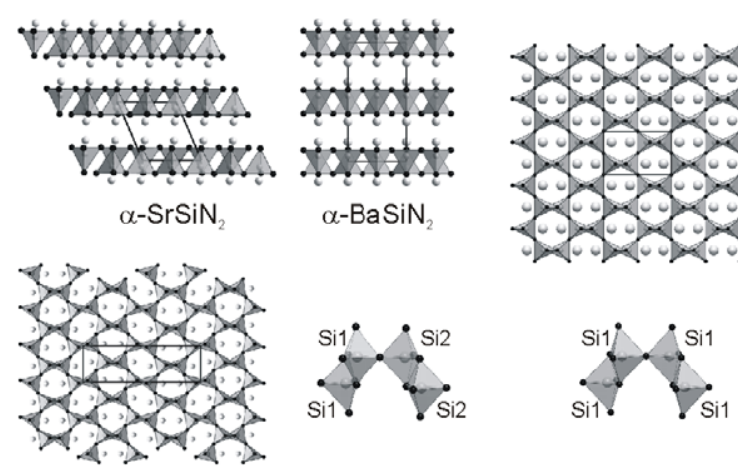

$\mathrm{Pbcm}$ structure type

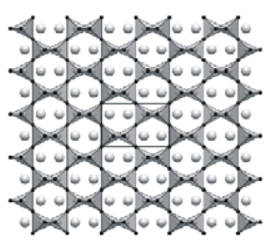

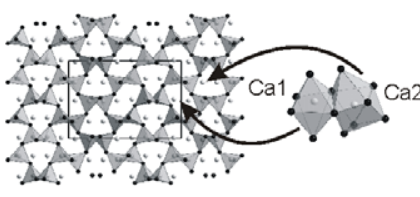

$\alpha-\mathrm{CaSiN}_{2}$
Imma structure type

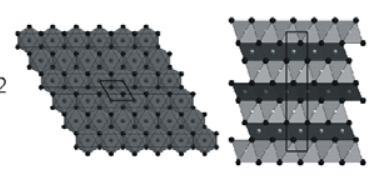

$m-\mathrm{LiFeO}_{2}$
Figure 1. Crystal structures of $\mathrm{SrSiN}_{2}$ and $\mathrm{BaSiN}_{2}$ polymorphs: (1) $\alpha-\mathrm{SrSiN}_{2}$, view along [010] $\left(\mathrm{SiN}_{4}\right.$ tetrahedra drawn light grey, $\mathrm{Sr}$ atoms between the $\mathrm{SiN}_{4}$ tetrahedra layers); (2) $\alpha-\mathrm{BaSiN}_{2}$, view along [001] $\left(\mathrm{SiN}_{4}\right.$ tetrahedra drawn light grey, Ba atoms between the $\mathrm{SiN}_{4}$ tetrahedra layers); (3) Imma structure; top, view along [001]; bottom, edge- and corner-sharing $\mathrm{SiN}_{5}$ trigonal bipyramids $\left(\mathrm{SiN}_{5}\right.$ trigonal bipyramids light grey, metal atoms in channels); (4) $\mathrm{Pbcm}$ structure; left, view along [001]; right, edge- and corner-sharing $\mathrm{SiN}_{5}$ trigonal bipyramids $\left(\mathrm{SiN}_{5}\right.$ trigonal bipyramids light grey, metal atoms in channels); (5) $\alpha$-CaSiN 2 , view along [100] $\left(\mathrm{SiN}_{4}\right.$ tetrahedra drawn light grey); (6) $\mathrm{m}-\mathrm{LiFeO}_{2}$ (left, octahedral layer, view along [001]; right, stacking of octahedral layers, view along [010]).

as early as $3 \mathrm{GPa}$. It is a transition from the monoclinic ground state of $\mathrm{SrSiN}_{2}$ (we assign the label $\alpha$ to this structure) to an orthorhombic high-pressure phase (denoted $\beta-\mathrm{SrSiN}_{2}$ ) with $\mathrm{BaSiN}_{2}$-type structure. Since both structures are closely related, this transition comes as no surprise. Due to the small enthalpy differences, some caution is advised, since the estimated transition pressure may be significantly influenced by further entropy contributions as well as methodological imperfections. Nevertheless, the smallness of the transition pressure should make it possible to realize the new compound in standard high-pressure equipment, including high-volume presses.

$\beta-\mathrm{SrSiN}_{2}$ remains the most stable polymorph up to $14 \mathrm{GPa}$, when $\gamma-\mathrm{SrSiN}_{2}$ comes into existence. $\gamma-\mathrm{SrSiN}_{2}$ adopts another orthorhombic structure (space group $\mathrm{Pbca}$, no 61) resembling that of $\mathrm{CaSiN}_{2}$. This structure, in which corner-sharing $\mathrm{SiN}_{4}$ tetrahedra form a three-dimensional network, can be derived from the idealized $\beta$-cristobalite structure through a D1-type distortion (figure 1) [2]. With $\mathrm{SrSiN}_{2}$ adopting the structure of the lower homologue $\mathrm{CaSiN}_{2}$ at high pressure, the system follows an unusual path. Typical in high-pressure science is the opposite trend, with many examples given in [11].

A third high-pressure modification, $\delta$-SrSiN 2 , will appear at $85 \mathrm{GPa}$. It adopts a hitherto unknown orthorhombic structure (space group $\mathrm{Pbcm}$ (no 57)), which results from a distortion of the $\mathrm{CaSiN}_{2}$ type (figure 1 ). $\delta-\mathrm{SrSiN}_{2}$ comprises $\mathrm{Si}$ in 

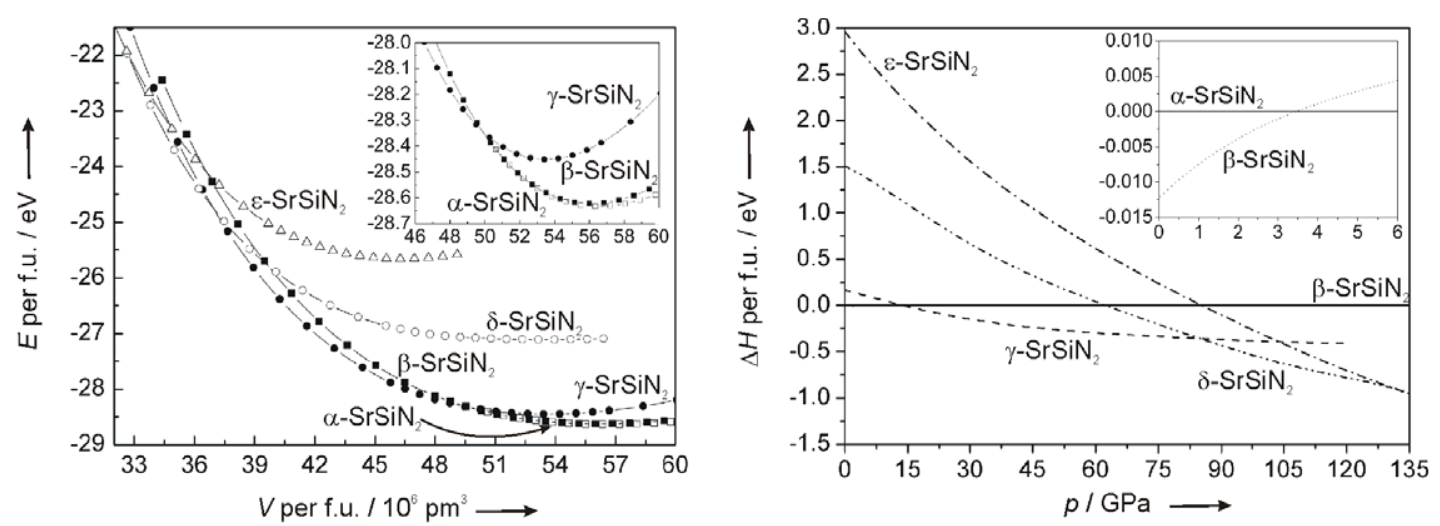

Figure 2. Left: energy-volume ( $E-V$ ) phase diagram of $\alpha-, \beta-, \gamma-, \delta$ - and $\varepsilon$ - $\mathrm{SrSiN}_{2}$, calculated within the GGA. Each symbol represents a calculation. Right: enthalpy-pressure $(H-p)$ diagram for the transition of $\alpha-\mathrm{SrSiN}_{2}$ into $\beta$-SrSiN $\mathrm{Sr}_{2}\left(p_{\mathrm{t}}=3 \mathrm{GPa}\right)$, into $\gamma-\mathrm{SrSiN} \mathrm{S}_{2}$ $\left(p_{\mathrm{t}}=14 \mathrm{GPa}\right)$, into $\delta-\mathrm{SrSiN}_{2}\left(p_{\mathrm{t}}=85 \mathrm{GPa}\right)$ and into $\varepsilon-\mathrm{SrSiN}_{2}\left(p_{\mathrm{t}}=131 \mathrm{GPa}\right)$ (derived from the evaluation of the $E-V$ data by the Murnaghan EOS)

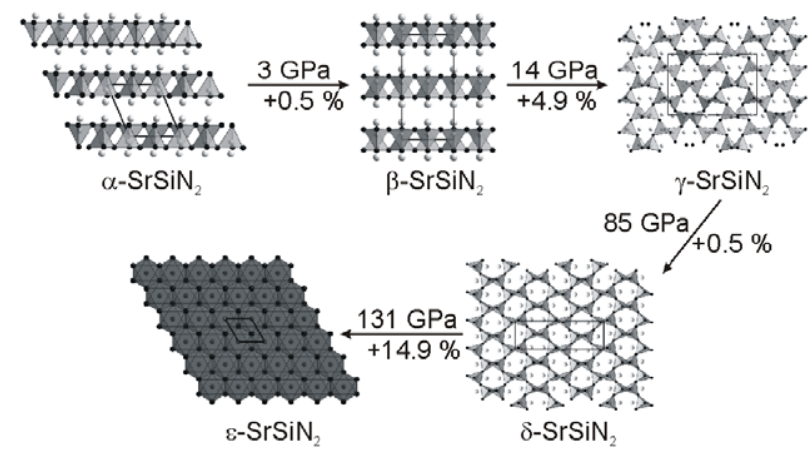

Figure 3. Sequence of structures of $\mathrm{SrSiN}_{2}$; transition pressures and density changes are indicated along the arrow.

trigonal bipyramidal coordination. It thus takes some $85 \mathrm{GPa}$ to increase the coordination of $\mathrm{Si}$ from four to five. $\mathrm{Sr}$ on the other hand remains eightfold coordinated in this structure, as it was previously in $\alpha-, \beta$ - and $\gamma-\mathrm{SrSiN}_{2}$. Finally, at pressures exceeding $131 \mathrm{GPa}$, a fourth modification, $\varepsilon-\mathrm{SrSiN}_{2}$, becomes the most favourable structure of $\mathrm{SrSiN}_{2} \cdot \varepsilon-\mathrm{SrSiN}_{2}$ is a superstructure of the $\mathrm{NaCl}$ type (an $\mathrm{m}-\mathrm{LiFeO}_{2}$ type with space group $R \overline{3} m$, no 166) [12]. Both $\mathrm{Si}$ and $\mathrm{Sr}$ are octahedrally coordinated (figure 1). A summary of the sequence of structures of $\mathrm{SrSiN}_{2}$ as pressure increases is shown in figure 3.

Zero-pressure energies, volumes and densities as well as bulk moduli of the five modifications of $\mathrm{SrSiN}_{2}$ are given in table 1. $\delta$-SrSiN 2 has the lowest bulk modulus (33 GPa) of all polymorphs, which renders it the most compressible among the $\mathrm{SrSiN}_{2}$ polymorphs. We attribute this finding to an exceptionally large compressibility of some $\mathrm{Si}-\mathrm{N}$ bonds in this structure.

\section{2. $\mathrm{BaSiN}_{2}$}

Our results of energy-volume calculations for different $\mathrm{BaSiN}_{2}$ polymorphs are shown in figure 4, which also includes the corresponding enthalpy-pressure diagram. The layered ground state modification $\alpha-\mathrm{BaSiN}_{2}$ with orthorhombic space group symmetry ( $\mathrm{Cmca}$, no 64 [2]) remains the most stable
Table 1. $E_{0}, V_{0}, B_{0}$ and $\rho_{0}$ for $\alpha-, \beta-, \gamma-, \delta$ - and $\varepsilon-\mathrm{SrSiN}_{2}$.

\begin{tabular}{lllll}
\hline & $\begin{array}{l}E_{0} / \text { f.u. } \\
(\mathrm{eV})\end{array}$ & $\begin{array}{l}V_{0} / \text { f.u. } \\
\left(10^{6} \mathrm{pm}^{3}\right)\end{array}$ & $B_{0}(\mathrm{GPa})$ & $\rho_{0}\left(\mathrm{~g} \mathrm{~cm}^{-3}\right)$ \\
\hline$\alpha-\mathrm{SrSiN}_{2}$ & -28.633 & 56.34 & 102 & 3.24 \\
$\beta-\mathrm{SrSiN}_{2}$ & -28.623 & 56.00 & 104 & 4.26 \\
$\gamma-\mathrm{SrSiN}_{2}$ & -28.452 & 53.41 & 125 & 4.47 \\
$\delta-\mathrm{SrSiN}_{2}$ & -27.110 & 53.15 & 33 & 4.49 \\
$\varepsilon-\mathrm{SrSiN}_{2}$ & -25.658 & 46.28 & 163 & 5.16 \\
\hline
\end{tabular}

Table 2. $E_{0}, V_{0}, B_{0}$ and $\rho_{0}$ for $\alpha-, \beta$ - and $\gamma-\mathrm{BaSiN}_{2}$.

\begin{tabular}{lllcl}
\hline & $\begin{array}{l}E_{0} / \text { f.u. } \\
(\mathrm{eV})\end{array}$ & $\begin{array}{l}V_{0} / \text { f.u. } \\
\left(10^{6} \mathrm{pm}^{3}\right)\end{array}$ & $B_{0}(\mathrm{GPa})$ & $\rho_{0}\left(\mathrm{~g} \mathrm{~cm}^{-3}\right)$ \\
\hline$\alpha-\mathrm{BaSiN}_{2}$ & -28.7114 & 62.50 & 89 & 5.14 \\
$\beta-\mathrm{BaSiN}_{2}$ & -28.2975 & 59.12 & 129 & 5.43 \\
$\gamma-\mathrm{BaSiN}_{2}$ & -26.699 & 55.40 & 135 & 5.80 \\
\hline
\end{tabular}

modification of $\mathrm{BaSiN}_{2}$ up to $41 \mathrm{GPa}$. At this pressure a $\mathrm{CaSiN}_{2}$-type modification (see figure 1 , labelled $\beta$-BaSiN ${ }_{2}$ ) will form. The three-dimensional network of corner-sharing $\mathrm{SiN}_{4}$ tetrahedra found in $\beta$-BaSiN 2 will then gradually distort into a structure comprising edge-sharing trigonal bipyramids. We denote this structure $\gamma-\mathrm{BaSiN}_{2}$, and it adopts the aforementioned $\mathrm{Pbcm}$ structure of $\delta-\mathrm{SrSiN}_{2}$ (figure 1). The transformation process is finished at a pressure of $105 \mathrm{GPa}$. Therefore, $\mathrm{BaSiN}_{2}$ follows a similar structural development as $\mathrm{SrSiN}_{2}$. However, an all-octahedral structure of $\mathrm{BaSiN}_{2}$ will not appear for pressures up to $150 \mathrm{GPa}$.

The complete sequence of high-pressure phases of $\mathrm{BaSiN}_{2}$ together with transition pressures and density changes is illustrated in figure 5. In table 2 we collect zero-pressure energies, volumes and densities as well as bulk moduli of the three modifications of $\mathrm{BaSiN}_{2}$.

For $\gamma-\mathrm{BaSiN}_{2}$ we encountered an interesting structural relation. There exists a path towards a more symmetric structure with space group Imma $\left(\gamma^{\prime}-\mathrm{BaSiN}_{2}\right.$; see figures 1 and 6). According to our calculations, $\gamma-\mathrm{BaSiN}_{2}$ adopts this higher symmetric structure at lower pressure. Note, however, that the Imma type of $\gamma^{\prime}-\mathrm{BaSiN}_{2}$ is a metastable structure, 

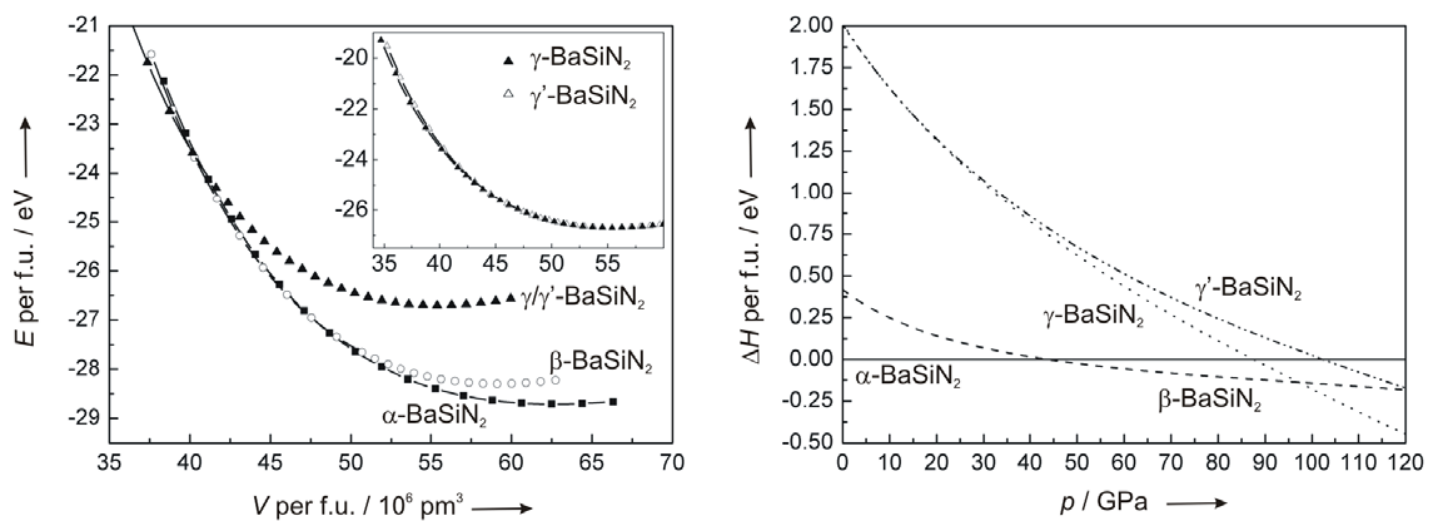

Figure 4. Left, energy-volume $(E-V)$ phase diagram of $\alpha-, \beta-, \gamma$-and $\gamma^{\prime}-\mathrm{BaSiN}_{2}$, calculated within the GGA. Each symbol represents a calculation. The inset shows the $E-V$ curves of $\gamma-\mathrm{BaSiN}_{2}$ and of $\gamma^{\prime}-\mathrm{BaSiN}_{2}$ (symmetry fixed to Imma). Right, enthalpy-pressure ( $H-p$ ) diagram for the transition of $\alpha-\mathrm{BaSiN}_{2}$ into $\beta-\mathrm{BaSiN}_{2}\left(p_{\mathrm{t}}=43 \mathrm{GPa}\right)$ and into $\gamma-\mathrm{BaSiN}_{2}\left(p_{\mathrm{t}}=105 \mathrm{GPa}\right)$ as well as of $\gamma^{\prime}-\mathrm{BaSiN}_{2}($ Imma $)$ into $\gamma-\mathrm{BaSiN}_{2}(\mathrm{Pbcm})\left(p_{\mathrm{t}}=32-35 \mathrm{GPa}\right)$.

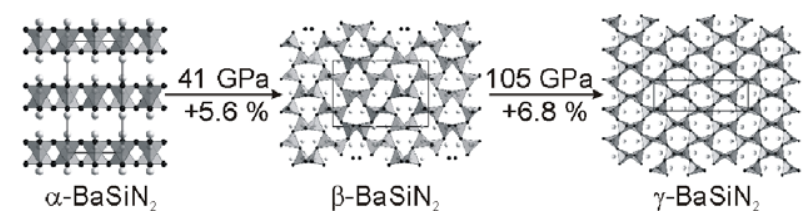

Figure 5. Sequence of structures of $\mathrm{BaSiN}_{2}$ including transition pressures and changes in density.

since $\beta$-and then at lower pressures $\alpha$ - $\mathrm{BaSiN}_{2}$ are even more favourable. Moreover, above $35 \mathrm{GPa}$ the lower-symmetry $\gamma-\mathrm{BaSiN}_{2}$ is favoured over $\gamma^{\prime}-\mathrm{BaSiN}_{2}$, if we constrain the symmetry of the latter. The tight structural relation between $\gamma$ - and $\gamma^{\prime}-\mathrm{BaSiN}_{2}$ (a comparison of atomic positions in the $\mathrm{Pbcm}$ and Imma structures is given in supplementary data available at stacks.iop.org/JPhysCM/21/275408), however, let us hypothesize that $\gamma-\mathrm{BaSiN}_{2}$ cannot be quenched to zero pressure. Instead, it may be that we observe a progressive distortion from the $P b c m$ structure to an Imma structure. The Imma structure of $\gamma^{\prime}-\mathrm{BaSiN}_{2}$, finally, optimizes upon further pressure reduction towards a low-density, low-coordinated structure.

\section{Discussion}

We propose four high-pressure phases of $\mathrm{SrSiN}_{2}$. The first transition occurs already as low as $3 \mathrm{GPa}$, from the monoclinic $\alpha-\mathrm{SrSiN}_{2}$ to a higher-symmetry orthorhombic structure. This transformation follows the pressure-homologue rule [13], according to which high-pressure conditions favour the formation of phases with the structure of heavier homologues. Presumably, this displacive phase transformation will have a low activation energy barrier, because only small displacements of the atoms (compare supplementary data available at stacks.iop.org/JPhysCM/21/275408) suffice to make the transformation (figure 7). Indeed, this will make a quenching of $\beta-\mathrm{SrSiN}_{2}$ a true challenge. Nevertheless, the orthorhombic $\beta$-phase of $\mathrm{SrSiN}_{2}$ should be detectable in in situ experiments, e.g. in the DAC.

Interestingly, the proposed transformation $\beta-\mathrm{SrSiN}_{2} \rightarrow$ $\gamma$ - $\mathrm{SrSiN}_{2}$ (with $\mathrm{CaSiN}_{2}$-type structure) then does not follow the aforementioned empirical 'rule'. Unlike so many silicate structures [11], the nitridosilicates $\mathrm{SrSiN}_{2}$ and $\mathrm{BaSiN}_{2}$ adopt the structure of a smaller homologue at high pressure. The nature of this phase transformation is unambiguously reconstructive: the connection pattern of $\mathrm{SiN}_{4}$ tetrahedra changes fundamentally, and a layered structure is converted into an extended, three-dimensional network.

Transforming $\gamma$ - into $\delta$-SrSiN 2 again is a displacive process. It follows a klassengleiche $(k 2)$ group-subgroup relation pathway from orthorhombic $\mathrm{Pbca}\left(\gamma-\mathrm{SrSiN}_{2}\right)$ to Pbcm $\left(\delta-\mathrm{SrSiN}_{2}\right)$ (cf figure 6 and supplementary data available at stacks.iop.org/JPhysCM/21/275408). Like the transformation from $\alpha$ - to $\beta-\mathrm{SrSiN}_{2}$, we can expect this

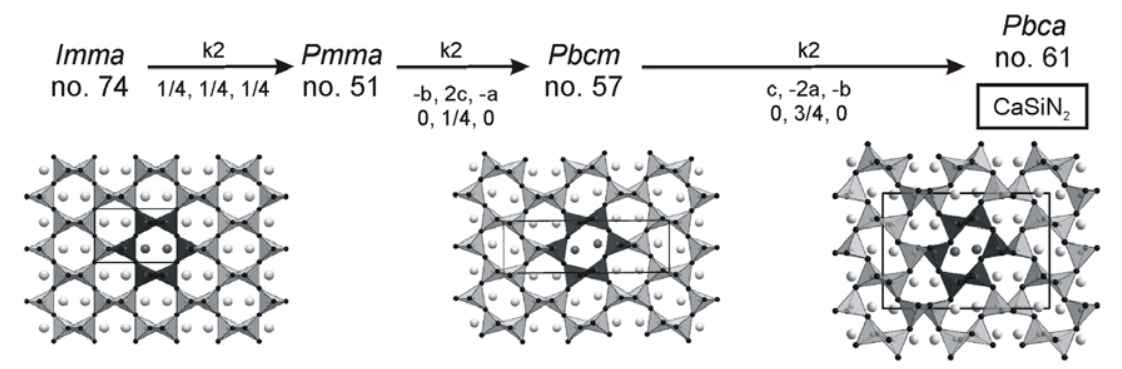

Figure 6. Illustrating the group-subgroup relation between the high-symmetry Imma structure, the $P b c m$ type, and $\mathrm{CaSiN}_{2}(P b c a)$. These structures may appear in the phase diagram of $\mathrm{BaSiN}_{2}$ as $\beta$ (right, $\mathrm{CaSiN}_{2}$ type), $\gamma$ (middle, Pbcm structure), and $\gamma^{\prime}-\mathrm{BaSiN}_{2}($ left, Imma structure). 


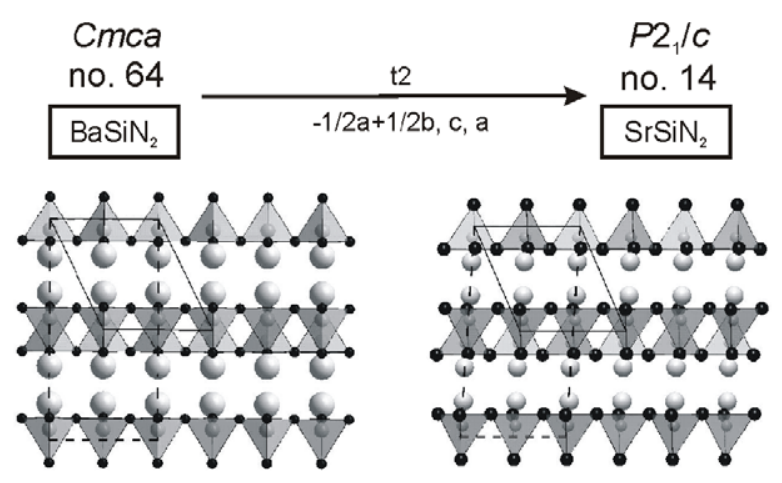

Figure 7. Group-subgroup relation of the $\mathrm{BaSiN}_{2}$ and the $\mathrm{SrSiN}_{2}$ structure. Unit cell drawn: (1) solid line, $P 2_{1} / c$; (2) dashed line, Cmca.

phase transformation to take place gradually. This time, however, we will observe an increase in the coordination number for $\mathrm{Si}$. Note that similar gradual coordination changes are encountered in $\mathrm{B}$-sesquioxide to $\mathrm{A}$-sesquioxide phase transitions frequently found for rare earth oxides $\mathrm{M}_{2} \mathrm{O}_{3}$. A small displacement of the atoms during the transition results in increased coordination numbers for the constituting atoms. These phase transformations are typically observed in situ only, as the A-sesquioxide structure often reverts to the Bsesquioxide structure upon pressure release [14]. Furthermore, it has to be noted that upon pressure reduction the $\mathrm{Si}-$ $\mathrm{N}_{a x}$ bonds of the $\mathrm{SiN}_{5}$ trigonal bipyramids in $\delta$-SrSiN 2 become too long (compare supplementary data available at stacks.iop.org/JPhysCM/21/275408). Therefore, it is most likely that this phase re-transforms into $\delta-\mathrm{SrSiN}_{2}$ or turns amorphous at lower pressures.

The fourth phase transition occurs at very high pressures (131 GPa), achieving a further increase of the coordination of $\mathrm{Si}$ from five to six. By adopting the $\mathrm{m}-\mathrm{LiFeO}_{2}$-type structure, a superstructure of the rock-salt structure type [12], $\varepsilon-\mathrm{SrSiN}_{2}$ finally follows the trend we have already discovered for $\mathrm{BeSiN}_{2}, \mathrm{MgSiN}_{2}$ and $\mathrm{CaSiN}_{2}$ [1].

$\mathrm{BaSiN}_{2}$ adheres to the same high-pressure structure sequence as $\mathrm{SrSiN}_{2}$. From orthorhombic Cmca $\mathrm{BaSiN}_{2}$ it transforms into a $\mathrm{CaSiN}_{2}$-type structure, followed by the displacive phase transformation into the $\mathrm{Pbcm}$-type structure (figure 6 and supplementary data available at stacks.iop.org/JPhysCM/21/275408). No transformation into the $\mathrm{m}-\mathrm{LiFeO}_{2}$-type structure was found up to $150 \mathrm{GPa}$. As we already noted, the $\mathrm{Pbcm}$ structure of $\gamma-\mathrm{BaSiN}_{2}$ may distort through a cascade of higher-symmetry structures into a very open structure with low density and high energy. Since the nature of the $\beta / \gamma$-phase transformation is also displacive, this process will compete with the re-transformation into $\beta$ - $\mathrm{BaSiN}_{2}$ upon pressure release. $\gamma$ - $\mathrm{BaSiN}_{2}$ may thus be observed in situ only.

\section{Summary}

We calculated the high-pressure phase diagram of $\mathrm{SrSiN}_{2}$ and $\mathrm{BaSiN}_{2}$. Our results let us propose several new modifications of $\mathrm{SrSiN}_{2}$ and $\mathrm{BaSiN}_{2}$ surpassing the layered ground state modifications upon compression. Ultimately, Si will exhibit coordination numbers higher than four in both systems. $\delta-\mathrm{SrSiN}_{2}$ and $\gamma-\mathrm{BaSiN}_{2}$ both exhibit $\mathrm{SiN}_{5}$ trigonal bipyramids, while $\varepsilon-\mathrm{SrSiN}_{2}$ even comprises $\mathrm{SiN}_{6}$ octahedra. Interestingly, both phase systems at one point violate the empirical pressure-homologue rule, as they both adopt a $\mathrm{CaSiN}_{2}$-type modification at high pressure. Furthermore, we describe an as-yet unknown $\mathrm{Pbcm}$-type high-pressure phase for both $\mathrm{SrSiN}_{2}$ and $\mathrm{BaSiN}_{2}\left(\delta-\mathrm{SrSiN}_{2}\right.$ and $\left.\gamma-\mathrm{BaSiN}_{2}\right)$. As all transition pressures are calculated to values below $150 \mathrm{GPa}$, there are good chances to observe the new phases in in situ DAC experiments. Some of the new phases can hopefully be quenched to ambient pressure.

\section{Acknowledgments}

Financial support by the Deutsche Forschungsgemeinschaft, DFG (priority programme SPP 1236, project SCHN 377/13 and KR 1805/10 and Heisenberg-programme Kr 1805/9), as well as the Fonds der Chemischen Industrie FCI, Germany, is gratefully acknowledged. The authors further would like to thank the Leibniz-Rechenzentrum, Munich, for computational resources on the Linux cluster system, as well as the Texas Advanced Computing Center at Austin.

\section{References}

[1] Römer S R, Kroll P and Schnick W 2009 J. Phys.: Condens. Matter 21275407

[2] Gál Z A, Mallinson P M, Orchard H J and Clarke S J 2004 Inorg. Chem. 433998

[3] Hohenberg P and Kohn W 1964 Phys. Rev. B 136864

[4] Kresse G and Hafner J 1993 Phys. Rev. B 47558

Kresse G and Hafner J 1994 Phys. Rev. B 4914251

Kresse G 1993 PhD Thesis Technische Universität Wien

Kresse G and Furthmüller J 1996 Comput. Mater. Sci. 615

Kresse G and Furthmüller J 1996 Phys. Rev. B 5411169

[5] Perdew J P 1991 in Electronic Structures of Solids ' 91 ed P Ziesche and H Eschrig (Berlin: Akademie Verlag)

[6] Kresse G and Joubert J 1999 Phys. Rev. B 591758

[7] Monkhorst H J and Pack J D 1976 Phys. Rev. B 135188

[8] Fachinformationszentrum Karlsruhe 2005-2008 Inorganic Crystal Structure Database Karlsruhe (Germany)

[9] Spek A L 2003 PLATON, A Multipurpose Crystallographic Tool (Utrecht: Utrecht University)

Spek A L 2003 J. Appl. Crystallogr. 367

[10] Murnaghan F D 1944 Proc. Natl Acad. Sci. USA 30244

[11] Liu L and Basset W A 1986 Elements, Oxides and Silicates. High-Pressure Phases with Implications for the Earth's Interior (Oxford: Oxford University Press)

[12] Douakha N, Holzapfel M, Chappel E, Chouteau G, Croguennec L, Ott A and Ouladdiaf B 2002 J. Solid State Chem. 163406

[13] Neuhaus A 1964 Chimia 1893

[14] Adachi G, Imanaka N and Kang Z C 2004 Binary Rare Earth Oxides (Berlin: Springer, Kluwer-Academic)

Guo Q, Zhao Y, Jiang C, Mao W L and Wang Z 2008 Solid State Commun. 145250

Hoekstra H R 1966 Inorg. Chem. 5754

Hoekstra H R and Gingerich K A 1964 Science 1461163

Husson E, Proust C, Gillet P and Itie J P 1999 Mater. Res. Bull. 342085

Hongo T, Kondo K-I, Kazutaka K G and Atou T 2007 J. Mater. Sci. 422582 